\title{
Lidil
}

Revue de linguistique et de didactique des langues

\section{La question de l'éthique en didactique des langues- cultures : aperçu historique et remarques prospectives}

The Issue of the Ethics in Language and Culture Didactics: Historical Overview and Prospective Remarks

\section{Emmanuel Antier}

\section{OpenEdition}

Journals

Édition électronique

URL : http://journals.openedition.org/lidil/4832

DOI : 10.4000/lidil.4832

ISSN : 1960-6052

\section{Éditeur}

UGA Éditions/Université Grenoble Alpes

\section{Édition imprimée}

ISBN : 978-2-37747-048-8

ISSN : $1146-6480$

\section{Référence électronique}

Emmanuel Antier, «La question de l'éthique en didactique des langues-cultures : aperçu historique et remarques prospectives », Lidil [En ligne], 57 | 2018, mis en ligne le 28 mars 2018, consulté le 30 avril 2019. URL : http://journals.openedition.org/lidil/4832 ; DOI : 10.4000/lidil.4832

Ce document a été généré automatiquement le 30 avril 2019

(C) Lidil 


\title{
La question de l'éthique en didactique des langues-cultures : aperçu historique et remarques prospectives
}

\author{
The Issue of the Ethics in Language and Culture Didactics: Historical Overview \\ and Prospective Remarks
}

Emmanuel Antier

\section{Introduction 1}

1 La question de l'éthique n'a jamais été autant d'actualité en didactique des languescultures (désormais DLC), à tout le moins dans le paysage didactique français. Alors que seuls deux numéros de revue lui avaient été consacrés avant les années 2010, on en recense actuellement cinq, ainsi qu'un ouvrage, qui, dans leur intitulé au moins, font une référence explicite à l'éthique. Pour plus de clarté, nous les citons ici dans l'ordre chronologique de leur parution :

- (1994). Les langues modernes, 3 : L'éthique.

- (1998). Études de linguistique appliquée, 109 : De l'éthique en didactique des langues étrangères.

- (2013). Recherche et pratiques pédagogiques en langues de spécialité, 32(2) : Quelle place pour l'éthique dans l'enseignement des langues de spécialité?

- (2013). BEACCO, Jean-Claude (dir.), Éthique et politique en didactique des langues. Autour de la notion de responsabilité, Paris : Didier.

- (2016). Les langues modernes, 4 : Éthique et enseignement des langues.

- (2017). Recherches et applications, 62 : Agir éthique en didactique du FLE/FLS.

2 Ce regain d'intérêt n'est pas sans rappeler celui que l'on observe en sciences de l'éducation, lesquelles ont vu émerger, ces dernières années, la question de l'éthique professionnelle des enseignants (voir p. ex. Moreau, 2012 ; Prairat, 2013 ; Lorius, 2015). 
Mais le parallèle s'arrête là. La mise en comparaison dans deux disciplines montre en effet assez clairement le caractère tâtonnant, confus, voire diffus de la réflexion sur l'éthique en DLC.

3 Afin d'illustrer cette idée, nous essaierons, dans un premier temps, de dresser un aperçu historique de la réflexion menée sur cette thématique en DLC. Il s'agira tout autant, dans une perspective descriptive, de rendre compte des caractéristiques de cette réflexion, que, dans une perspective normative, de la questionner à l'aune des apports conceptuels de la philosophie morale et des sciences de l'éducation. À travers cette démarche, nous posons l'hypothèse que ces deux disciplines peuvent apporter une contribution significative pour penser la problématique de l'éthique en DLC.

4 Sur la base des constats effectués au terme de ce parcours historique, nous essaierons, dans un second temps, de formuler quelques propositions concrètes en vue de penser la question de l'éthique professionnelle des enseignants de langue-culture. Deux pistes seront principalement explorées. La première nous mènera à réfléchir aux moyens de développer une éthique appliquée de l'enseignement en DLC; la seconde nous conduira à défendre l'idée d'une éthique minimale dans la formation des enseignants.

Au total, il s'agira d'examiner la question de l'éthique en DLC, d'une manière aussi bien rétrospective que prospective, afin d'essayer de la clarifier et, ce faisant, de la constituer comme un véritable champ de recherche.

\section{Aperçu historique}

6 Un rapide examen des travaux menés sur la thématique de l'éthique en DLC permet d'identifier deux mouvements successifs, auxquels on peut attribuer deux préoccupations différentes. Un premier, qui débute dans le courant des années 1980, correspond à l'émergence d'une réflexion déontologique. Un second, amorcé dans les années 2000 avec la publication du Cadre européen commun de référence pour les langues (Conseil de l'Europe, 2001, désormais CECR), est marqué par la promotion de l'éducation plurilingue et interculturelle. À travers l'examen de ces deux mouvements - ou plutôt, comme nous le verrons, de ces deux moments successifs d'un mouvement continu -, voyons donc plus précisément comment la question de l'éthique a été posée jusqu'à présent en DLC.

\subsection{Une éthique domaniale}

7 La question de l'éthique en DLC est d'abord intimement liée à la recherche d'autonomie de la discipline. En se définissant comme une discipline d'intervention, la DLC a été amenée à se questionner sur la dimension morale de son intervention. Sous l'impulsion notamment de Galisson, Puren et Forestal s'est développée une réflexion sur la responsabilité sociale des acteurs du domaine. En 1986, Galisson regrette ainsi l'absence de réflexion déontologique et en appelle consécutivement à la prise en compte des questions éthiques dans l'enseignement-apprentissage des langues :

[...] toutes les disciplines qui engagent l'avenir de l'homme ont besoin d'une « déontologie ». Chacun sait, par exemple, que la médecine a la sienne [...] mais, dans notre domaine, tout se passe comme si n'importe qui pouvait enseigner n'importe quelle langue et n'importe quelle culture, à n'importe qui d'autre, n'importe comment, et n'importe où. Or, quantité de problèmes se posent aux enseignants de langues, aux décideurs, à la collectivité tout entière, qui relèvent de 
la simple conscience ou de la morale politique et ne sauraient être ni éludés ni laissés au hasard, ni même réglés au coup par coup. (p. 48) réflexion éthique dans le processus d'autonomie et de légitimation de la DLC :

Cette discipline a d'autant plus d'intérêt à remplir ses obligations éthiques qu'elles constituent des marques de spécificité précieuses pour son image : comme toutes les disciplines d'intervention, son action engage sa responsabilité morale et c'est du respect des règles qu'elle se donne que nait le respect qu'on lui porte. (p. 84)

Malgré ces appels à la prise en compte de la dimension éthique de l'enseignementapprentissage des langues-cultures, peu de recherches ont été consacrées à cette question. Par ailleurs, les quelques écrits disponibles sur cette thématique, principalement ceux de Forestal $(2001,2004,2007)$ sont prioritairement centrés sur la conceptualisation d'une éthique domaniale. Dans une perspective interventionniste, il s'agit davantage de réfléchir à la responsabilité sociale de l'enseignant-chercheur qu'à celle de l'enseignant-praticien. La réflexion est orientée vers la défense de valeurs particulières. Ce passage, extrait de la conclusion d'un article de Forestal (2007), illustre cette conception engagée de l'éthique en DLC :

Face à la montée inquiétante des violences, spécialement dans les milieux des jeunes, nous avons souhaité insister sur la nécessité d'inverser cette tendance périlleuse par une formation à une compétence éthique en DLC. Celle-ci devrait contribuer à donner des repères en matière de valeurs (leurs contradictions ou leurs complémentarités) et aider également à refonder un enseignement laïc et républicain. (p. 31, en italique dans le texte)

Il est à noter que ce discours sur l'éthique, fondé sur une vision particulière du bien, trouve en partie sa source dans le positionnement de Galisson. Son engagement critique contre un " économisme uniformisateur et retors » l'a ainsi amené à développer l'idée d'une « éducation aux langues-cultures »:

Pour souligner l'incompatibilité de l'éducation et de l'économie, pour faire valoir le caractère hautement éducatif des langues-cultures, pour marquer notre appartenance et baliser notre territoire, pour donner du lustre à l'éthique, pour la sacraliser, pour la rendre plus présente, plus circulante dans les DI [Disciplines d'intervention], je suggère, quand le contexte le permet et en milieu institutionnel au moins, de substituer «Éducation aux langues-cultures " à la vieillissante appellation « Enseignement/apprentissage de langues-cultures ». (1998, p. 89)

Plus récemment, et pour mieux marquer encore l'idée du primat de l'« éducation » sur celui de l'«enseignement-apprentissage», Galisson (2002), dans un article intitulé "Didactologie: de l'éducation aux langues-cultures à l'éducation par les languescultures ", a plaidé pour une éducation dans laquelle l'objet langue-culture constitue non pas la fin mais le moyen de l'éducation.

En mettant l'accent sur la dimension axiologique des langues-cultures, en faisant des valeurs la finalité de leur enseignement, et en prônant une éducation qui relève de la « composante morale de l'individu » (ibid., p. 504), Galisson a probablement ouvert la voie à une instrumentalisation de la DLC. Paru en 1997, le numéro de la revue Études de linguistique appliquée intitulé « Éduquer pour une Europe des langues-cultures? " annonce d'ailleurs assez clairement les prémices de la pensée - aujourd'hui dominante - qui vise à mettre la DLC au service du projet politique européen. 


\subsection{L'influence du Conseil de l'Europe}

13 Si le Conseil de l'Europe (désormais CdE) est actif dans l'enseignement des langues depuis les années 1960, son implication politique dans ce domaine date plus précisément des années 1990 avec la promotion de la "communication interculturelle» dans le but de développer une «citoyenneté démocratique». Au cours des années 2000, cette orientation s'est renforcée avec l'affirmation d'une politique linguistique centrée sur la promotion de l'éducation plurilingue et interculturelle (désormais EPI), éducation formalisée notamment dans le CECR et largement promue depuis dans les divers textes et outils mis en ligne sur le site du $\mathrm{CdE}^{2}$.

14 En quoi consiste donc cette éducation? Quelles sont ses finalités? Et surtout, dans la perspective qui nous préoccupe ici, comment contribue-t-elle à formaliser la question de l'éthique en DLC? Ces quelques lignes extraites de L'éducation plurilingue et interculturelle comme projet (Conseil de l'Europe, 2009) offrent un premier éclaircissement à notre questionnement :

En fait l'éducation plurilingue et interculturelle se caractérise d'abord par ses finalités qui concernent, avant tout, les droits fondamentaux de chaque apprenant et qui se fondent sur des valeurs destinées à assurer sa formation en tant qu'individu et que citoyen. Ces valeurs sont constituées par les principes directeurs des actions du Conseil de l'Europe: la cohésion et la solidarité sociales, la démocratie participative, la compréhension réciproque ainsi que le respect et la valorisation de la diversité linguistique et culturelle. L'éducation plurilingue et interculturelle, orientée par de telles valeurs, est à mettre en œuvre par leur pleine intégration dans la philosophie qui oriente le projet que l'École se donne, dans la posture éthique de chaque enseignant et, de façon opérationnelle, dans les approches et les méthodologies adoptées. (p. 7-8; nous soulignons.)

En première analyse, on constate que l'EPI s'adresse tout autant aux apprenants ( ( formation en tant qu'individu et que citoyen ») qu'aux enseignants (« dans la posture éthique de chaque enseignant»), et qu'elle comporte une forte dimension morale et politique ( la cohésion et la solidarité sociales, la démocratie participative, la compréhension réciproque ainsi que le respect et la valorisation de la diversité linguistique et culturelle»). Ce constat rejoint celui dressé par Maurer (2011) qui a montré comment, avec l'EPI, la formation linguistique a été progressivement minorée au profit d'une éducation morale.

16 Cette situation peut apparaitre comme relevant d'un paradoxe, voire d'une ironie de l'histoire. En effet, si la question de l'éthique en DLC a été initialement posée avec l'arrivée à maturité de cette discipline et sa revendication à l'autonomie, elle semble désormais avoir été récupérée, sous la forme d'une éducation morale, dans un projet politique externe à la DLC, celui de la formation d'un citoyen européen. Nous rejoignons ici l'interrogation de Springer (2015) :

Ces nouveaux enjeux politiques européens ne peuvent qu'avoir un impact fort sur la DDL [Didactique des langues]. La question est de savoir si l'on n'est pas en train de changer de dépendance en passant d'une dépendance scientifique à une dépendance politique et idéologique. Cet impact, pour la DDL, en tant que discipline, aboutit-il à une consolidation/émancipation ou à une reconfiguration imposant une autre dépendance? (p. 261)

Springer (2015) laisse la question ouverte en rappelant toutefois que « la DDL n'est pas la propriété des chercheurs»(p.265). Cette remarque est importante. Sur le plan de 
l'éthique, nous pourrions risquer une réponse plus tranchée. En s'éloignant de la classe de langue et des problèmes éthiques tels qu'ils se posent dans la pratique, en promouvant une éducation par les langues-cultures, c'est-à-dire une éducation dans laquelle, on l'a vu, les langues-cultures ne constituent qu'un moyen en vue d'une fin à définir, il semble bien que les didacticiens aient progressivement laissé les experts politologues ou sociolinguistes s'emparer de la problématique éthique en DLC.

\subsection{Bilan} constats sur l'état actuel de la recherche sur l'éthique en DLC. Premièrement, même si la question de l'éthique - ou à tout le moins celle des "valeurs »- apparait comme centrale en DLC, notamment avec le développement de l'EPI, aucune étude ne s'appuie explicitement sur les apports conceptuels de la philosophie morale ou sur les avancées des recherches menées en sciences de l'éducation. Tout se passe en effet comme si le sens des concepts liés à l'éthique n'avait pas besoin d'être précisé, comme si les théories morales, pourtant disponibles, n'avaient pas besoin d'être convoquées. Dans ces conditions, il est à craindre que les discours sur l'éthique sombrent dans une forme de verbiage moralisateur.

Cette crainte nous apparait comme d'autant plus fondée que l'EPI vise ouvertement à la promotion d'un bien personnel. Un tel projet repose sur une conception maximaliste de la morale, c'est-à-dire une conception basée, entre autres, sur l'idée de perfectionnisme, d'exemplarité et d'épanouissement personnel. Les lignes d'ouverture du CECR (2001) exposent assez clairement cette orientation vertuiste :

Dans une approche interculturelle, un objectif essentiel de l'enseignement des langues est de favoriser le développement harmonieux de la personnalité de l'apprenant et de son identité en réponse à l'expérience enrichissante de l'altérité en matière de langue et de culture. Il revient aux enseignants et aux apprenants eux-mêmes de construire une personnalité saine et équilibrée à partir des éléments variés qui la composeront. (p. 9 ; nous soulignons.)

Or, il y a des raisons de s'opposer à cette conception maximaliste de la morale en DLC. Nous avons déjà dénoncé ailleurs l'aporie d'une éducation basée sur l'enseignement de savoir-être, c'est-à-dire sur la modification du rapport des apprenants avec eux-mêmes (Antier, 2017a). Face au risque de dérive moraliste et ethnocentriste d'un tel programme éducatif, nous avons choisi d'endosser et de défendre une conception plus minimaliste de morale, laquelle implique, notamment, de rester neutre à l'égard des différentes conceptions du bien personnel ou de la vie « réussie $»^{3}$.

21 Le deuxième constat issu de notre aperçu historique porte sur le manque de recherche empirique. Même si quelques études sont disponibles sur cette problématique, aucune n'a été directement menée à ce jour sur la thématique de l'éthique professionnelle des enseignants de langue-culture. Les travaux sur ce thème s'intéressent en effet davantage, on l'a vu, à l'éthique de l'enseignant-chercheur qu'à celle de l'enseignant-praticien. Leur visée est majoritairement normative, politiquement engagée. Il ne s'agit pas, dans une perspective descriptive, de conceptualiser les enjeux éthiques de l'agir enseignant, mais bien plutôt, dans une perspective prescriptive, de promouvoir le rôle sociétal et la contribution politique de la DLC. 
D’une manière générale, les questions de terrain ont été largement délaissées jusqu’à présent. Et il y a des raisons de le regretter. Nous l'avons vu, Springer (2015), par exemple, considère que la DLC « vit grâce aux enseignants et aux élèves sur le terrain pédagogique » (p. 265). Ce positionnement rappelle celui de Puren. Dans un article où il montre comment cette discipline s'est historiquement constituée - depuis la perspective méthodologique jusqu'à la perspective didactologique en passant par la perspective didactique -, Puren (1999) insiste sur l'importance du « niveau » méthodologique :

Le niveau méthodologique est indispensable dans la perspective d'une gestion démocratique de la discipline didactique, c'est-à-dire qui prenne en compte la dignité égale de ses différents acteurs et l'intérêt a priori égal de leurs contributions communes. Je sais qu'un certain nombre de mes collègues universitaires m'accusent sur ce point de démagogie, et un nombre encore plus certain d'inspecteurs d'anarchisme, mais je persiste et signe : le niveau méthodologique est le seul niveau où tout «simple» enseignant peut contester à partir de sa seule expérience pratique individuelle l'autorité de tout spécialiste quel qu'il soit (psychologue, sociologue, historien, spécialiste de littérature ou de civilisation, linguiste, inspecteur... ou didacticien): "Désolé, vos théories et vos propositions sont officielles / sont sans doute scientifiques, mais je constate que dans mes classes leur mise en œuvre serait/est impossible, ou trop couteuse, ou inefficace. » (p. 39)

Ce raisonnement nous semble pouvoir s'appliquer pareillement à la question de l'éthique. D'après l'épistémologie complexe développée et défendue par Puren en DLC, il importe de relier les trois perspectives constitutives de cette discipline, de pouvoir constamment passer de l'une à l'autre selon un principe de récursivité. Même si, par définition, les questions éthiques relèvent d'une mise en perspective didactologique, elles peuvent et doivent récursivement être reliées à la perspective méthodologique, laquelle constitue le centre de la discipline. Cette conception est en accord avec l'idée d'une réflexion sur l'éthique professionnelle des enseignants - telle qu'elle se développe actuellement en sciences de l'éducation -, c'est-à-dire d'une éthique centrée sur les acteurs de l'enseignement-apprentissage, et non pas théorisée de l'extérieur, selon une logique de l'expertise et de la spécialisation institutionnelle.

\section{Remarques prospectives pour la formation des enseignants}

Comment donc penser la question de l'éthique en DLC? Comment en finir avec les tentatives de théorisation externe? Comment sortir du piège que représente une conception perfectionniste de la morale? Sur la base de ce questionnement, nous essaierons à présent de formuler quelques remarques prospectives en vue de penser ou de repenser la formation à l'éthique professionnelle des enseignants de langue-culture.

\subsection{En finir avec la logique de l'expertise}

Dans la section précédente, nous avons vu que la question de l'éthique en DLC, initialement posée avec l'arrivée à maturité de cette discipline, a été récupérée, sous la forme d'une éducation morale, dans le projet politique européen. Progressivement, les experts du CdE ont investi cette thématique dans une perspective prescriptive, surplombante, éloignée de la pratique des enseignants. 

sur le terrain. Nous avons vu notamment que l'idée d'une éthique centrée sur les acteurs de l'enseignement-apprentissage était en accord avec l'épistémologie complexe défendue par Puren en DLC, ainsi que plus généralement avec l'idée d'une éthique appliquée de l'enseignement, laquelle, précisons-le, ne consiste pas à administrer d'en haut une théorie normative à une pratique mais, au contraire, à mettre en évidence la manière dont une pratique particulière peut questionner les théories normatives.

dans le seul domaine de la DLC. Elle nous est directement inspirée par la réflexion menée en sciences de l'éducation, réflexion qui distingue généralement deux phases: l'acquisition d'un savoir formel en philosophie morale, et l'entrée dans un processus d'analyse éthique des pratiques. Dans ce développement, nous nous intéresserons plus précisément à cette seconde phase.

29

fréquemment convoqué pour développer la capacité d'analyse des futurs praticiens (voir p. ex. Prairat, 2013). Typiquement, le formateur propose à ses étudiants de réfléchir sur des dilemmes ou des conflits moraux, c'est-à-dire des situations problématiques pour lesquelles il existe au moins deux solutions possibles. Pour plus de précisions, nous reproduisons ici la méthode en six étapes élaborée par Jeffrey (2015) :

1) présenter en ses mots ce qui pose problème du point de vue de l'éthique ;

2) faire ressortir les valeurs, normes et devoirs professionnels concernés ;

3) colliger les informations essentielles ;

4) présenter au moins deux solutions possibles avec les avantages et les inconvénients ;

5) défendre avec des arguments la solution retenue ;

6) dire ce qui aurait pu être fait pour prévenir le problème. (p. 36)

Toute situation se présentant sous la forme d'un dilemme ayant trait aux rapports de l'enseignant à ses apprenants, à ses collègues, à l'institution d'emploi, ou encore à l'enseignement-apprentissage (choix didactiques, méthode d'évaluation, etc.) peut ainsi servir de point de départ à l'analyse des pratiques. Invités à se projeter dans une situation problématique, les futurs enseignants s'entrainent à délibérer en examinant, d'une part, les valeurs en jeu dans leur décision et, d'autre part, les conséquences possibles de cette décision sur les personnes concernées. L'accent est mis sur le débat collectif, sur le dialogue entre pairs, sur la capacité à expliciter sa position et à prendre en compte celle des autres.

Il faut, écrit Prairat, organiser le travail sur les dilemmes par petits groupes de pairs stables pour permettre le passage de la délibération au dialogue et du dialogue à la délibération. [...]. Le dialogue est moral non seulement en tant qu'il se rapporte à des questions d'ordre moral mais aussi et surtout parce qu'il est un échange entre partenaires qui manifestent un authentique souci de l'autre et de la relation qui les 
unit. Il s'agit, en somme, de délibérer au sein d'un groupe qui dialogue. (2013,

p. 218)

31 On le voit, ce qui importe lors de cette phase d'analyse, c'est moins le produit de la délibération que le processus de réflexion. La formation à l'éthique à travers des études de cas ne vise pas à la formulation de solutions définitives, mais à sensibiliser les futurs praticiens aux enjeux éthiques de leur pratique, à leur permettre de les prendre en charge eux-mêmes. On s'éloigne ici assez radicalement d'une formation pensée selon la logique externe de l'expertise, potentiellement culpabilisatrice parce qu'inadaptée à la réalité du terrain. En donnant aux enseignants les moyens de structurer leur réflexion, une telle formation vise au contraire à soutenir leur responsabilité, à les outiller pour faire face à la complexité de leur pratique.

\subsection{Vers la reconnaissance du pluralisme moral}

Du point de vue de l'éthique, nous pouvons situer la complexité de l'agir enseignant dans le pluralisme moral, c'est-à-dire dans la pluralité des repères moraux disponibles. Comment donc penser une formation à l'éthique dans un monde marqué par le pluralisme des valeurs et des conceptions du bien?

la première partie de notre étude, nous avons vu que l'EPI constituait actuellement la seule option disponible pour penser la formation à l'éthique professionnelle des enseignants de langue-culture. Recourant à la distinction entre morale maximale et morale minimale, nous avons souligné, par ailleurs, que cette éducation relevait d'une conception maximaliste de la morale fondée notamment sur l'idée de perfectionnisme.

Pour les défenseurs d'une éthique minimale (voir notamment Walzer, 1994 ; Ogien 2007), plus une conception de la morale est maximaliste, plus elle cherche à régenter nos manières d'être, et plus sa portée est relative à une société donnée. Inversement, plus une conception de la morale est minimaliste, plus elle se réduit au principe de non-nuisance à autrui, et plus grandes sont les chances qu'elle ait une portée universelle. D'où l'hypothèse selon laquelle le minimalisme constitue une option morale plus en phase avec le pluralisme qui caractérise les sociétés modernes.

Sur la base de cette hypothèse, il importe de réfléchir aux implications du minimalisme dans le cadre d'une formation à l'éthique en DLC. Nous en évoquerons deux. De nouveau, elles nous sont inspirées par les sciences de l'éducation. La première, développée par Lorius (2015), suppose de rejeter l'idée selon laquelle l'éducation a des objectifs. La seconde, due à Prairat (2013), porte sur l'exigence de neutralité. Voyons donc brièvement en quoi consistent ces deux implications du minimalisme dans la formation des enseignants.

Si elle peut sembler à priori contre-intuitive, la proposition selon laquelle l'éducation n'a pas d'objectif repose toutefois sur la conjonction de trois arguments solidement établis. Premièrement, écrit Lorius (2015), "l'éducation, comme toutes les facettes de la vie humaine, est d'abord caractérisée par la chance ${ }^{4} »($ p. 66) ; deuxièmement, elle est « une imprégnation, une action du milieu sur les nouveaux arrivants » (p. 67) ; troisièmement, elle «se fixe des fins qui, par définition, sont référées à l'avenir, lequel, est inconnu et donc incertain » (p. 67). Consécutivement, Lorius appelle à "considérer avec prudence une quelconque toute puissance éducative » (p. 68) :

L'éducation, écrit-il, doit donc être comprise comme un horizon, une utopie, dont

l'objet même est de s'inscrire hors d'une volonté de toute puissance par rapport au 
devenir des individus dont elle a la charge : c'est à cette condition qu'elle sera un projet et non un programme. (p. 68) dravail novateur sur la déontologie enseignante, Prairat (2013) a formalisé un minimalisme déontologique qu'il fait reposer sur trois grandes règles : la sobriété normative, le souci de la stabilité et l'exigence de neutralité. Arrêtons-nous brièvement sur cette troisième règle, l'exigence de neutralité, qui ordonne de rester silencieux sur les raisons de l'engagement professionnel et sur la figure du maitre idéal.

Toute déontologique, écrit Prairat, qui se hasarderait à énoncer les «bonnes raisons » d'entrer dans le métier discréditerait de facto certains professionnels qui, sans démériter comme le montre à l'envi l'expérience, ont choisi cette activité professionnelle pour des raisons prosaïques, voire accidentelles. De même, la promotion de la figure du maitre idéal contredit non seulement l'évidence selon laquelle l'excellence peut prendre plusieurs formes, mais, aussi et surtout, elle empêche de promouvoir l'idée de pratiques fiables, pratiques toujours accessibles et qui, précisément, ne s'encombrent d'aucune figure idéale. (2013, p. 180)

De nouveau, il nous faut constater que cette implication minimaliste entre en opposition avec la conception perfectionniste de l'EPI, laquelle tend à proposer, comme figure normative, l'image d'un enseignant idéal, lui-même promoteur de l'EPI. Cette conception est clairement perceptible, par exemple, dans le projet de définir la « posture éthique de chaque enseignant» (Conseil de l'Europe, 2009, p.8). On la trouve aussi dans des formulations telles que :

Il faut aussi que les enseignants adhèrent aux objectifs d'enseignement/ apprentissage qui sont proposés dans le CARAP, au travers des descripteurs de compétences et de ressources, qu'ils soient persuadés de leur utilité et de leur légitimité. (Candelier \& De Pietro, 2012, p. 49, en italique dans le texte.)

Ou encore :

L'enseignant en langues doit être préparé à développer des compétences de communication plurilingues et pluriculturelles [...]. Un objectif aussi ambitieux ne peut être atteint sans formation complète, pouvant être divisée en deux axes inséparables l'un de l'autre : une dimension sociale et personnelle et une dimension professionnelle. (Conseil de l'Europe, 2007, p. 16)

En résumé, à l'encontre de cette conception maximaliste, le minimalisme moral en formation des enseignants implique, d'une part, de renoncer à donner des objectifs absolus à l'éducation et, d'autre part, de rompre avec un vertuisme moralisateur prônant la figure d'un.e enseignant.e idéal.e. 


\subsection{Vers une formation complexe à l'éthique}

41 En ce qu'elle propose de rester neutre sur les différentes conceptions de la vie bonne, l'éthique minimale, avons-nous dit, s'accorde particulièrement bien avec le pluralisme qui caractérise les sociétés modernes. Elle nous semble aussi en phase avec l'idée d'une formation complexe, telle que l'a développée Puren en DLC à la fin des années 1990. C'est en tout cas la perspective que nous nous proposons d'explorer dans ce dernier développement.

Constatant, d'une part, la fin des méthodologies constituées, et considérant, d'autre part, l'éclectisme méthodologique comme une réponse pragmatique donnée par les enseignants à la complexité didactique, Puren (1999) en a appelé à un changement de modèle formatif en DLC :

Une formation méthodologique moderne, écrit-il, est une formation qui prend en compte le niveau didactique; ce qui veut dire qu'elle ne privilégie pas a priori telle ou telle approche à l'exclusion des autres [...], mais qu'elle vise à donner aux enseignants les moyens de sélectionner, hiérarchiser et articuler eux-mêmes différemment un maximum d'approches variées en fonction de la variation de leurs objectifs, de leurs supports, des situations d'enseignement/apprentissage... et de leurs élèves. (p. 33)

Une telle proposition, prenant en compte la complexité des situations didactiques, se veut en rupture avec le modèle formatif généralement mis en œuvre qui consiste, à l'inverse, à simplifier la complexité.

Dans ce modèle, précise Puren, former un enseignant, c'est le former à utiliser la dernière méthodologie ou approche en vigueur. Modèle très pernicieux en formation initiale, puisqu'ainsi on ne donne pas aux enseignants les moyens de faire face à toute la complexité des situations de classe et de la problématique didactique; et qu'on leur propose des systématisations qui sont impossibles à mettre en œuvre dans la pratique, avec toutes les frustrations et culpabilisations que cela peut provoquer. (2001, p. 14)

Ces remarques nous semblent pouvoir être appliquées avec pertinence au projet d'une formation à l'éthique. Ainsi, nous pouvons considérer que, de la même manière qu'une formation méthodologique ne devrait pas privilégier à priori une méthodologie à l'exclusion des autres, une formation à l'éthique ne devrait pas, non plus, privilégier à priori une option morale particulière. Une telle conception de la formation à l'éthique suggère quelques implications fortes qu'il convient d'examiner.

Premièrement, nous l'avons vu, l'EPI apparait actuellement comme l'unique option morale disponible dans le discours didactique. On peut dès lors poser l'hypothèse que les enseignants de langue-culture, ainsi que leurs formateurs, en quête de repères moraux et de sens à donner à leur pratique, auront tendance à adopter ipso facto la conception morale de l'EPI. Ce qui pose problème dans le cadre d'un tel modèle formatif, c'est l'absence d'alternative et, consécutivement, l'incapacité d'une telle formation à épouser la complexité éthique de l'agir enseignant. Une formation complexe devrait permettre aux enseignants d'acquérir, à l'inverse, des repères moraux pluriels, en accord avec l'hétérogénéité constitutive de leur pratique.

Deuxièmement, la proposition d'une formation complexe conduit à poser la question de savoir si l'EPI constitue une option morale recevable. En d'autres termes, devons-nous endosser ici l'idée qu'une formation à l'éthique puisse intégrer des repères maximalistes ? 
Pour répondre à cette question, il convient de bien distinguer ce qui est (le factuel) de ce qui doit être (le normatif). La défense d'une éthique minimale relève d'un positionnement normatif, donc discutable. D'un point de vue factuel, la recherche de Lorius (2014) a montré, notamment, que les enseignants mobilisent des repères moraux variés, allant du minimalisme au maximalisme. La question qui se pose alors n'est donc plus de savoir si le maximalisme constitue une option morale recevable, mais bien plutôt de savoir quel en serait l'intérêt, pour quel contexte et dans quelle situation. De nouveau, le problème que pose ici l'EPI, c'est sa prétention à formaliser l'ensemble des situations éthiques ou, à tout le moins, son incapacité à envisager ses propres limites en fonction de la variété des contextes. Or, il y a des raisons de penser que son intérêt est, par exemple, extrêmement limité, voire inexistant, dans un contexte multiculturel d'enseignement-apprentissage (Antier, 2017b, chap. 6), c'est-à-dire un contexte marqué par la pluralité des conceptions de l'acte éducatif et de la vie « réussie ».

Troisièmement - et nous finirons sur cette idée générale -, la perspective d'une formation complexe à l'éthique en DLC suppose le développement de recherches menées dans une perspective empirique. Elle implique, une nouvelle fois, de rompre avec la logique de l'expertise, et de mieux comprendre la manière particulière dont se pose la problématique de l'éthique dans la pratique des enseignants.

\section{Conclusion}

Notre étude a poursuivi deux objectifs conjoints : celui, d'abord, de dresser un aperçu historique de la réflexion menée sur la question de l'éthique en DLC; et celui, ensuite, de formuler quelques propositions en vue de développer cette réflexion. Notre analyse historique nous a permis de montrer que la question de l'éthique en DLC repose traditionnellement sur une conception maximaliste de la morale, sur un projet politique éloigné de la pratique réelle des enseignants.

Contre le monisme de l'EPI, et contre la logique de l'expertise caractéristique des travaux $\mathrm{du} \mathrm{CdE}$, nous avons finalement proposé l'idée d'une formation complexe à l'éthique, laquelle suppose une diversification des options morales disponibles en DLC et, consécutivement, le développement de recherches menées dans une perspective empirique. En accord avec l'idée d'une éthique appliquée, nous pensons en effet que ce n'est qu'à l'aune de telles recherches qu'il sera possible de proposer des options morales en phase avec l'hétérogénéité constitutive de l'agir enseignant.

On l'aura compris, en l'état actuel de la réflexion sur l'éthique en DLC, penser la formation à l'éthique des enseignants de langue-culture, c'est avant tout essayer de sortir du piège que représente une conception moraliste et paternaliste de l'acte éducatif. En matière d'éthique, plus que dans d'autres domaines encore, il nous semble urgent de résister aux sirènes de la grandiloquence, aux discours incantatoires et déconnectés de l'expérience morale des enseignants. 


\section{BIBLIOGRAPHIE}

ANTIER, Emmanuel. (2017a). La problématique éthique du « savoir-être » en didactique des langues-cultures : quelques réflexions autour du CARAP et autres productions du Conseil de l'Europe. Revue TDFLE, 70. Disponible en ligne sur <http://revue-tdfle.fr/13-les-numeros-de-larevue-tdfle/tdfle-numero-70/25-la-problematique-ethique-du-savoir-etre-en-didactique-deslangues-cultures-quelques-reflexions-autour-du-carap-et-autres-productions-du-conseil-de-leurope> (consulté le 21 novembre 2017).

ANTIER, Emmanuel. (2017b). L'éthique professionnelle des enseignants de langue-culture en contexte multiculturel : constats, enjeux, et perspectives (Thèse de doctorat non publiée). Université Paul Valéry - Montpellier 3, Montpellier.

CANDELIER, Michel \& De PiETRo, Jean-François. (2012). Le CAPAP - Une introduction à l'usage. Strasbourg : Conseil de l'Europe.

CONSEIL DE L'EUROPE. (2001). Un cadre européen commun de référence pour les langues : apprendre, enseigner, évaluer. Apprentissage des langues et citoyenneté européenne. Paris : Didier.

CONSEIL DE L'EUROPE. (2007). La dimension plurilingue et pluriculturelle dans la formation des enseignants de langues : kit de formation. Strasbourg : Conseil de l'Europe.

CONSEIL DE L'EUROPE. (2009). L'éducation plurilingue et interculturelle comme projet. Strasbourg :

Conseil de l'Europe.

FORESTAL, Chantal. (2001). Du droit et du devoir d'une discipline à exister par elle-même : la sage utopie d'un être de passion. Études de linguistique appliquée, 123, 485-490.

FORESTAL, Chantal. (2004). Et si l'on parlait d'incivilité institutionnelle et d'immobilisme ? Études de linguistique appliquée, 133, 9-26.

ForESTAL, Chantal. (2007). La dynamique conflictuelle de l'éthique pour une compétence éthique en DLC. Études de linguistique appliquée, 145, 111-123.

GALISSON, Robert. (1986). Éloge de la didactologie/didactique des langues et des cultures (maternelles et étrangères). Études de linguistique appliquée, 64, 39-54.

GALISSON, Robert. (1998). À la recherche de l'éthique dans les disciplines d'intervention. Études de linguistique appliquée, 109, 83-127.

GALISSON, Robert. (2002). Didactologie : de l'éducation aux langues-cultures à l'éducation par les langues-cultures. Études de linguistique appliquée, 128, 497-510.

JEFFREY, Denis. (2015). Enseigner l'éthique aux futurs enseignants. Dans L.-A. Saint-Vincent (dir.), Le développement de l'agir éthique chez les professionnels en éducation. Formations initiale et continue (p. 25-42). Québec : Presses de l'Université du Québec.

LORIUS, Vincent. (2014). Éduquer dans un monde pluraliste. Le minimalisme moral au secours de l'école républicaine ? Recherches en Éducation, h.-s. 6, 91-102.

LORIUS, Vincent. (2015). Le courage d'éduquer. Imagination morale et activité des éducateurs en contexte scolaire. Nancy : Presses universitaires de Nancy. 
MAURER, Bruno. (2011). Enseignement des langues et construction européenne : le plurilinguisme, nouvelle idéologie dominante. Paris : Archives contemporaines.

MEIRIEU, Philippe. (2015). Frankenstein pédagogue (9e éd.). Issy-les-Moulineaux : ESF.

Michaud, Yves. (2009). Qu'est-ce que le mérite? Paris : Bourin.

MOREAU, Didier (dir.). (2012). L'éthique professionnelle des enseignants : enjeux, structures et problèmes. Paris : L'Harmattan.

OGIEN, Ruwen. (2007). L'éthique aujourd'hui : maximalistes et minimalistes. Paris : Gallimard.

PRAIRAT, Eirick. (2013). La morale du professeur. Paris : Presses universitaires de France.

PUREN, Christian. (1999). La didactique des langues-cultures étrangères entre méthodologie et didactologie. Les langues modernes, 3, 26-41.

PUREN, Christian. (2001). La problématique de la formation dans le contexte actuel de l'éclectisme méthodologique. Le nouveau bulletin de l'ADEAF, 78, 6-18.

SPRINGER, Claude. (2015). Et si la didactique des langues faisait fausse route ? Dans J.-M. Defays et coll. (dir.), Transversalités. 20 ans de FLES (vol. 1, p. 251-268). Bruxelles : EME éditions.

WALZER, Michael. (2004). Morale maximale, morale minimale (C. Fort, trad.). Paris : Bayard. (Ouvrage original publié en 1994 sous le titre Thick and Thin: Moral Argument at Home and Abroad, Notre Dame : University of Notre Dame Press).

\section{NOTES}

1. Cet article est issu de notre thèse de doctorat: L'éthique professionnelle des enseignants de langueculture en contexte multiculturel : constats, enjeux et perspectives, soutenue le 12 septembre 2017 sous la direction de Bruno Maurer, Université Paul Valéry - Montpellier 3.

2. Ces ressources sont consultables à l'adresse suivante: < www.coe.int/t/dg4/linguistic/ default_fr.asp>.

3. Sur la distinction entre minimalisme et maximalisme en philosophie morale, on se reportera utilement à L'éthique aujourd'hui : maximalistes et minimalistes (Ogien, 2007).

4. Pour étayer son argument, Lorius se réfère à Michaud selon qui «la chance constitutive est propre à l'individu, non pas au sens où il serait chanceux, mais au sens où il bénéficie d'une meilleure santé, de données génétiques meilleures, où il nait dans une famille mieux ou moins bien lotie » (Michaud, 2009, cité par Lorius, 2015, p. 66).

\section{RÉSUMÉS}

Dans cet article, on se propose d'abord de dresser un aperçu historique de la question de l'éthique en didactique des langues-cultures. Ce cheminement nous conduit à constater que l'éducation plurilingue et interculturelle promue par le Conseil de l'Europe constitue, à ce jour, la seule option morale disponible pour penser l'éthique professionnelle des enseignants de langueculture. Or, en ce qu'elle repose sur l'idée de perfectionnement personnel, cette éducation 
correspond à une conception maximaliste de la morale. Face au risque de dérive moraliste d'une telle visée éducative, nous prônons une conception plus minimaliste de l'éthique dans la formation des enseignants de langue-culture. Contre la logique de l'expertise caractéristique des travaux du Conseil de l'Europe et contre le monisme de l'éducation plurilingue et interculturelle, nous proposons finalement l'idée d'une formation complexe à l'éthique.

The purpose of this paper is first to provide an historical overview of the issue of the ethics in language and culture didactics. Our analysis leads us to recognize that the plurilingual and intercultural education promoted by the Council of Europe has now grown to be a dominant moral choice in language and culture didactics. However, inasmuch as it is based on the notion of personal development, this education represents a maximalist conception of morality. Faced with the drift into excessive moralism of such an educational aim, we advocate a more minimalist conception of ethics in the language and culture of teachers training. In opposition to the system of expert assessment which is typical of the Council of Europe's work, and contrary to the monism of plurilingual and intercultural education, we put forward the idea of complex ethical training.

\section{INDEX}

Mots-clés : didactique des langues-cultures, éthique, éducation plurilingue et interculturelle, formation des enseignants

Keywords : language and culture didactics, ethics, plurilingual and intercultural education, teacher training

\section{AUTEUR}

\section{EMMANUEL ANTIER}

Département de langue et littérature françaises de l'université de Fukuoka (Japon) 